\title{
EXTENSION OF ALGEBRAIC THEORIES
}

\author{
E. JAMES PEAKE AND GALEN R. PETERS
}

\begin{abstract}
The algebraic theories of Lawvere are extended in a natural way to small complete categories. These categories exhibit not only the operations and identities, but some of the homomorphisms, functions, objects and constructions which are encountered when working within the algebraic categories associated with the theories. The category of extended theories is isomorphic to the original category of theories. As an illustration, the extended theory of groups is used to construct commutator subgroups.
\end{abstract}

1. Introduction. In his dissertation [1], Lawvere characterizes "equationally definable classes of algebras" by means of "algebraic categories." In this paper an algebraic theory will be extended so that it exhibits not only the operations and identities, but some of the homomorphisms, functions, objects and constructions which are encountered when working within the algebraic category associated with the theory. For example, considering the theory of groups, there are associated with each group the following entities: its commutator subgroup, the quotient of the group modulo its commutator subgroup, the natural homomorphisms associated with the above groups, the free group on the underlying set of the group, and the set inclusion of the group into the free group. These entities are induced in a natural way by the theory of groups and it is possible to extend the theory of groups to encompass them.

In $\S 2$, a small complete category $\boldsymbol{A}_{\boldsymbol{\theta}}$, called an extended theory, will be constructed from an algebraic theory $\boldsymbol{A}$ in a manner such that every morphism of $\boldsymbol{A}_{\boldsymbol{\theta}}$ will be induced in a natural way by morphisms of the theory $\boldsymbol{A}$. The category of extended theories $\boldsymbol{T}_{\theta}$ will be isomorphic to the category of theories $\boldsymbol{T}$.

As an illustration, in $\S 3$, the structure of the extended theory of groups will be used to construct commutator subgroups.

The notation and basic concepts used in this paper are patterned after those used by Lawvere in [2]. Complete will always mean small complete, both left and right. If $A$ and $B$ are categories, $\operatorname{Cat}(A, B)$ will denote the category whose morphisms are the natural transformations between

Received by the editors May 25, 1971.

AMS 1970 subject classifications. Primary 18C10; Secondary 08A25, 18C05, 18 A30.

Key words and phrases. Algebraic theory, structure, semantics, algebraic category.

(c) American Mathematical Society 1972 
functors from $A$ to $B$. $\left(A^{b}, S\right)$ and $\left(A^{b}, A^{b}\right)$ will be skeletal subcategories of $\operatorname{Cat}\left(A^{b}, S\right)$ and $\operatorname{Cat}\left(A^{b}, A^{b}\right)$ respectively. It will be assumed that the $n$-fold products $\left(U_{A}\right)^{n}$ and $\operatorname{Id}^{n}$ are in $\left(A^{b}, S\right)$ and $\left(A^{b}, A^{b}\right)$ respectively for all $n=0,1,2, \cdots$, where Id is the identity functor. For $F_{A}$, the left adjoint of $U_{A}$, and an object $C$ in $\left(A^{b}, S\right)$, the composition $F_{A} C$ is identified with the object in $\left(A^{b}, A^{b}\right)$ to which it is isomorphic. Similarly, if $C$ and $D$ are objects in $\left(A^{b}, S\right)$ and $\left(A^{b}, A^{b}\right)$ respectively, $C D$ is considered to be in $\left(A^{b}, S\right)$. St and Sm denote the structure and semantics functors respectively.

2. Extended theories. Let $\boldsymbol{A} \in|\boldsymbol{T}|$ be given, where $|\boldsymbol{T}|$ is the class of objects of $\boldsymbol{T}$. Let $I: A \rightarrow A$ be the identity functor. Define $I^{n}: A \rightarrow A$ to be the $n$-fold product of $I$. Suppose $\beta: A^{k} \rightarrow A^{l}$ is in $A$. Define $\beta^{\#}: I^{k} \rightarrow I^{l}$ such that $\beta^{\#}$ at $A^{n}$ is $\beta^{n}$. If $\beta^{\#}$ is a natural transformation, a natural transformation $\beta^{\prime \prime}$ is induced from $\operatorname{Id}^{k}$ to $\operatorname{Id}^{l}$ where $\left(\beta^{\prime \prime}\right)_{f}$ at $A^{n}$ is $f\left(\beta^{n}\right)$.

If $\alpha: C \Rightarrow U_{A} K$ is in $\left(A^{b}, S\right)$ for some $K$ in $\left(A^{b}, A^{b}\right)$, a corresponding morphism $\alpha^{\prime}: F_{A} C \Rightarrow K$ is induced in $\left(A^{b}, A^{b}\right)$. Precisely, if $\varphi$ is the natural bijection from hom $\left(C f, U_{A} K f\right)$ into hom $\left(F_{A} C f, K f\right)$, then $\left(\alpha^{\prime}\right)_{f}=\varphi\left(\alpha_{f}\right)$ for each $f \in\left|\boldsymbol{A}^{b}\right|$.

THEOREM. Let $\boldsymbol{A}$ be an algebraic theory. There exists a category $\boldsymbol{A}_{\boldsymbol{\theta}}$ which is the smallest subcategory of $\left(\boldsymbol{A}^{b}, S\right)$ containing $\boldsymbol{A}$ such that there exists a subcategory $\boldsymbol{B}_{\theta}$ of $\left(\boldsymbol{A}^{b}, \boldsymbol{A}^{b}\right)$ satisfying:

(a) for each $\alpha \in \boldsymbol{A}$ such that $\alpha^{\#}$ is a natural transformation, $\alpha^{\prime \prime}$ is in $\boldsymbol{B}_{\theta}$;

(b) for each $\alpha \in \boldsymbol{A}_{\theta}, F_{A} \alpha$ is in $\boldsymbol{B}_{\theta}$;

(c) for each $\beta \in \boldsymbol{B}_{\theta}, U_{A} \beta \in \boldsymbol{A}_{\theta}$;

(d) for each $\alpha \in \boldsymbol{A}_{\theta}$ such that the codomain of $\alpha$ is $U_{A} K$ for some $K \in\left|\boldsymbol{B}_{\theta}\right|$, $\alpha^{\prime}$ is in $\boldsymbol{B}_{\theta}$;

(e) $\boldsymbol{A}_{\theta}$ and $\boldsymbol{B}_{\theta}$ are complete.

Proof. An ordinal induction will be used to construct $\boldsymbol{A}_{\boldsymbol{\theta}}$. Since $\boldsymbol{A}$ is isomorphic to $\mathrm{St} U_{A}$, which is a subcategory of $\left(A^{b}, S\right), A$ will be identified with St $U_{A}$. Defining $U_{0}$ to be St $U_{A}$, let $V_{0}$ be the completion of $U_{0}$ in $\left(\boldsymbol{A}^{b}, \boldsymbol{S}\right)$. Define $W_{0}$ to be the completion in $\left(\boldsymbol{A}^{b}, \boldsymbol{A}^{b}\right)$ of the category generated by all $\beta^{\prime \prime}$ as constructed above. Then define $U_{1}$ to be the category generated in $\left(A^{b}, S\right)$ by $U_{A}\left(W_{0}\right)$ and $V_{0}$ where $U_{A}\left(W_{0}\right)$ is the category obtained by composing the functor $U_{A}$ with objects in $W_{0}$.

Assuming that $U_{k}$ and $W_{l}$ for all $l \in k$ have been defined for a nonzero ordinal $k$, let $V_{k}$ be the completion of $U_{k}$ in $\left(A^{b}, S\right)$. Define $W_{k}$ to be the completion in $\left(A^{b}, A^{b}\right)$ of the category generated by $F_{A}\left(V_{k}\right), \cup\left\{W_{l}: l \in k\right\}$, and the class of all $\alpha^{\prime}$ as constructed above where $\alpha$ is in $V_{k}$ and $K$ is in $\bigcup\left\{W_{l}: l \in k\right\}$. Define $U_{k+1}$ to be the category generated in $\left(A^{b}, S\right)$ by $U_{A}\left(W_{k}\right)$ and $V_{k}$. For a limit ordinal $k$, define $U_{k}=\bigcup\left\{U_{l}: l \in k\right\}$. Let $\boldsymbol{A}_{\theta}=\bigcup\left\{U_{k}: k\right.$ is an ordinal $\}$ and $\boldsymbol{B}_{\theta}=\bigcup\left\{W_{k}: k\right.$ is an ordinal $\}$. 
Note that $\boldsymbol{A}_{\boldsymbol{\theta}}$ is a complete category. If only a finitely complete category were desired, a similar process could be used to obtain a category $\boldsymbol{A}_{\boldsymbol{\omega}}$, where $\omega$ is the first limit ordinal.

The following lemma will be used to define the categories $\boldsymbol{T}_{\boldsymbol{\theta}}$ and $\left(\boldsymbol{A}_{\boldsymbol{\theta}}\right)^{b}$.

LeMma [3, p. 143]. Let $M$ be a full subcategory of a category $C$, $K: M \rightarrow C$ be the insertion functor and $P_{c}: K / c \rightarrow M$ be the projection from the comma category $K / c$ where $c$ is an object of $M$. If $T: M \rightarrow D$ is a functor such that the composite $T P_{c}$ has a colimit in $D$ for each $c \in|C|$, then there exists a functor $S: C \rightarrow D$, unique up to natural isomorphism, such that $S K=T$ and the identity natural transformation $1: T \Rightarrow S K$ is part of a universal arrow $\langle S, 1\rangle$ from $T$ into $D^{K}$.

In the above lemma, $D^{K}: \operatorname{Cat}(C, D) \rightarrow \operatorname{Cat}(M, D)$ is defined by $D^{K}(\beta)=$ $\beta K$. Also note that the morphisms of $K / c$ are commutative triangles $g \circ K h=f$ in $C$ with $f: K m \rightarrow c$ and $g: K n \rightarrow c$ as domain and codomain respectively where $h: m \rightarrow n$.

If $\boldsymbol{X} \in|\boldsymbol{T}|$, let $I_{x}: X \rightarrow \boldsymbol{X}_{\boldsymbol{\theta}}$ be the inclusion embedding. Given $f: \boldsymbol{A} \rightarrow \boldsymbol{E}$ in $\boldsymbol{T}$, let $M=A, C=A_{\theta}, T=I_{B} f, D=E_{\theta}$, and $K=I_{A}$. Since $\boldsymbol{E}_{\theta}$ is complete and $I_{A} / c$ is small for all $c \in\left|A_{\theta}\right|, I_{B} f_{\circ} P_{c}$ has a colimit in $\boldsymbol{E}_{\theta}$. Hence the lemma implies that there exists $f_{\theta} \equiv S: \boldsymbol{A}_{\theta} \rightarrow \boldsymbol{E}_{\theta}$ such that $f_{\theta} K=I_{B} f$. The category $\boldsymbol{T}_{\theta}$ is defined to be the category of all objects $\boldsymbol{A}_{\theta}$ and functors $f_{\theta}$ obtained from $\boldsymbol{T}$ by the above process. The association of $f_{\theta}$ with $f$ defines a functor $\boldsymbol{F}: \boldsymbol{T} \rightarrow \boldsymbol{T}_{\boldsymbol{\theta}}$ which is an isomorphism, i.e., there exists another functor $F^{\prime}: T_{\theta} \rightarrow T$ such that $F F^{\prime}$ and $F^{\prime} F$ are identity functors.

For $f: A \rightarrow S$ in $A^{b}$, the lemma can be applied with $M=A, C=A_{\theta}, T=f$, $D=S$, and $K=I_{A}$ to obtain a unique functor $f_{\theta} \equiv S: A_{\theta} \rightarrow S$. Because the natural transformation $1_{f}: T \Rightarrow S K$ is part of a universal arrow $\left\langle S, 1_{f}\right\rangle$ from $f$ into $D^{K}, \alpha: f \Rightarrow g$ in $A^{b}$ induces a unique $\alpha_{\theta}: f_{\theta} \Rightarrow g_{\theta}$ in $\left(A_{\theta}, S\right)$ such that $D^{K}\left(\alpha_{\theta}\right) \circ 1_{f}=1_{g} \circ \alpha$. Hence the category $\left(A_{\theta}\right)^{b}$ is defined to be the subcategory of $\left(\boldsymbol{A}_{\theta}, S\right)$ obtained from $\boldsymbol{A}^{b}$ by the above process. $\boldsymbol{A}^{b}$ and $\left(\boldsymbol{A}_{\theta}\right)^{b}$ are isomorphic, and if they are identified in the construction of Lawvere's comma category (Cat, $S)$, then $\left(F^{\mathrm{op}} \mathrm{St}, \mathrm{Sm} F^{\prime \mathrm{op}}, \varphi^{\prime}\right):(\mathrm{Cat}, S) \rightarrow\left(\boldsymbol{T}_{\theta}\right)^{\mathrm{op}}$ is an adjunction where $F^{\prime \mathrm{op}}:\left(\boldsymbol{T}_{\theta}\right)^{\mathrm{op}} \rightarrow T^{\mathrm{op}}$ and $F^{\mathrm{op}}: T^{\mathrm{op}} \rightarrow\left(T_{\theta}\right)^{\mathrm{op}}$ are obtained from the isomorphisms $F^{\prime}: \boldsymbol{T}_{\theta} \rightarrow \boldsymbol{T}$ and $F: \boldsymbol{T} \rightarrow \boldsymbol{T}_{\theta}$ and $\varphi^{\prime}$ is the obvious extension of $\varphi$ in the adjunction (St, Sm, $\varphi):($ Cat, $\boldsymbol{S}) \rightarrow \boldsymbol{T}^{\mathrm{op}}$.

The original algebraic theories of Lawvere have now been extended to exhibit, not only the operations and identities, but natural consequences of them. Moreover, the method used maintains the structure of the original theories. The original morphisms between theories emerge when the extended theory morphisms are restricted to the original theories. Also the St-Sm adjunction is preserved. Hence the constructions have enriched the theories without destroying their original properties. 
3. Illustration. Let $\boldsymbol{A}$ be the theory of groups. There are operations $h, 0: A \times A \rightarrow A$ such that for any group $g$ and $(x, y)$ in $g(A) \times g(A)$, $g(h)(x, y)=x y x^{-1} y^{-1}$ and $g(0)(x, y)$ is the identity. The projection $p: g \Rightarrow$ $g /[g]$, where $p_{A}(x)=x[g(A)]$ and $[g]$ is the commutator subgroup, is in some sense the "group coequalizer" of $h$ and 0 , i.e., it is a factor of every homomorphism $q$ out of $g$ such that $g h=q 0$. It is not necessarily the set coequalizer of $h$ and 0 . There are no morphisms in the algebraic theories of groups or sets, or even in the extended theory of sets which correspond to the homomorphism $p$. However, the extended theory of groups contains such a morphism.

In general, given an algebraic theory $A$, there exists the functor $U \equiv U_{A}$ : $\boldsymbol{A}^{b} \rightarrow \boldsymbol{S}$. For each pair $(\alpha, \beta)$ in $\operatorname{hom}\left(U^{n}, U\right) \times \operatorname{hom}\left(U^{n}, U\right)$, consider all natural transformations $v: \mathrm{Id} \Rightarrow G$ where $\mathrm{Id}: A^{b} \rightarrow A^{b}$ is the identity functor and $G$ is arbitrary, with the property that $(U v) \alpha=(U v) \beta . U v$ is defined such that $(U v)_{x}=U\left(v_{x}\right)$ for all $x \in|A|^{b}$.

Call the class of these natural transformations $U(\alpha, \beta)$. A natural transformation $\lambda \in U(\alpha, \beta)$ will be called a special coequalizer of $\alpha$ and $\beta$ if given any natural transformation $v \in U(\alpha, \beta)$ there exists a unique natural transformation $\tau \in\left(\boldsymbol{A}^{b}, \boldsymbol{A}^{b}\right)$ such that $\tau \lambda=\nu$.

THEOREM. For each pair $(\alpha, \beta) \in \operatorname{hom}\left(U^{n}, U\right) \times \operatorname{hom}\left(U^{n}, U\right)$, there exists a special coequalizer $\lambda$ of $\alpha$ and $\beta$.

Proof. Given $\alpha, \beta: U^{n} \Rightarrow U$, consider $\alpha^{\prime}, \beta^{\prime}: F_{A} U^{n} \Rightarrow$ Id as constructed in $\S 2$. Let $c\left(\alpha^{\prime}, \beta^{\prime}\right): \operatorname{Id} \Rightarrow C\left(\alpha^{\prime}, \beta^{\prime}\right)$ be the coequalizer of $\left(\alpha^{\prime}, \beta^{\prime}\right)$ in the category $\left(\boldsymbol{A}^{b}, \boldsymbol{A}^{b}\right)$. Note that $U\left(c\left(\alpha^{\prime}, \beta^{\prime}\right)\right)$ appears in $U_{1} \subset \boldsymbol{A}_{\theta}$. It can be checked that $\lambda \equiv c\left(\alpha^{\prime}, \beta^{\prime}\right)$ is a special coequalizer of $\alpha$ and $\beta$.

Note that $\lambda$ in the existence theorem is unique up to isomorphism.

Let $\boldsymbol{A}$ be the theory of groups. There exists a functor $F: \boldsymbol{A}^{b} \rightarrow \boldsymbol{A}^{b}$ and a natural transformation $p: I d \Rightarrow F$ such that for $g \in\left|A^{b}\right|, F(g)=g /[g]$ and $p_{g}$ is the projection. It was stated previsously that $p_{g}$ was in some sense the "group coequalizer" of the two operations $h$ and 0 . It will be proved that $p$ is the special coequalizer of $h$ and 0 .

$\boldsymbol{A}$ is identified with $\mathrm{St} \operatorname{Sm} \boldsymbol{A}$ by the isomorphism $\eta: \boldsymbol{A} \rightarrow \mathrm{St} \mathrm{Sm} \boldsymbol{A}$ where $\eta(h)_{f}=f(h)$ for any $h \in \boldsymbol{A}$ and $f \in\left|\boldsymbol{A}^{b}\right|$. In particular, this identifies $h: A \times A \rightarrow A$ with $\eta(h): U_{A} \times U_{A} \Rightarrow U_{A}$. It can be shown that $\left(U_{A} p\right) \eta(h)=\left(U_{A} p\right) \eta(0)$. Now suppose there exists a $\tau: \operatorname{Id} \Rightarrow G$ such that $\left(U_{A} \tau\right) \eta(h)=\left(U_{A} \tau\right) \eta(0)$. Since $\left(p_{f}\right)_{A}$ is surjective for each $f \in\left|\boldsymbol{A}^{b}\right|$, it is possible to define a unique function $\left(\sigma_{f}\right)_{A}: F(f)(A) \rightarrow G(f)(A)$ such that $\left(\sigma_{f}\right)_{A} U_{A} \tau=\left(U_{A} p\right) f .\left(\sigma_{f}\right)_{A}$ can be extended to a homomorphism $\sigma_{f}: F(f) \Rightarrow G(f)$. Since $f$ was arbitrary, a natural transformation $\sigma$ is defined such that $\sigma p=\tau$. Thus $p$ is a special coequalizer of the pair $(\eta(h), \eta(0))$ which is identified with $(h, 0)$. Note that $U_{A} p \in \boldsymbol{A}_{\theta}$. 
If $\boldsymbol{A}$ is the algebraic theory of groups, both $p$ and $\delta: \operatorname{Id} \Rightarrow F$ appear in $B_{\theta}$, where $(\delta)_{f}$ is the trivial homomorphism from $f$ to $f /[f]$ for all $f \in\left|A^{b}\right|$. $\delta=p e^{\prime \prime} u$ where $e$ represents the zero-ary group operation and $u: \mathrm{Id}_{\Rightarrow} \Rightarrow \mathrm{Id}^{0}$. Thus the functor $F^{\prime}: A^{b} \rightarrow A^{b}: g \rightarrow[g]$, which is an equalizer of $p$ and $\delta$, appears in $\left|\boldsymbol{B}_{\theta}\right|$.

The construction of the commutator subgroup from the operations suggests that there are many other natural algebraic entities which may be consequences of the theory, some well known and others not extensively studied. The extended theories might well be useful in answering questions about specific algebras.

\section{REFERENCES}

1. F. W. Lawvere, Functorial semantics of algebraic theories, Unpublished Ph.D. Thesis, Columbia University, New York, 1963.

2. - Some algebraic problems in the context of functorial semantics of algebraic theories, Reports of the Midwest Category Seminar. II, Springer, Berlin, 1968, pp. 4161. MR 38 \#210.

3. S. Mac Lane, Categorical algebra, National Science Foundation Advanced Science Seminar, Bowdoin College, Mathematics Dept., Brunswick, Maine, 1969.

Department of Mathematics, Iowa State University, Ames, Iowa 50010

Current address (Peters): Department of Mathematics, Greenville College, Greenville, Illinois 62246 